\title{
Effect of Maternal Care on Hearing Onset Induced by Developmental Changes in the Auditory Periphery
}

\author{
Shana Adise, ${ }^{1}$ Aminat Saliu, ${ }^{1}$ Natalia Maldonado, ${ }^{2}$ Vivek Khatri, ${ }^{1}$ Luis Cardoso, ${ }^{2}$ and Adrián Rodríguez-Contreras ${ }^{1}$ \\ Departments of ${ }^{1}$ Biology and ${ }^{2}$ Biomedical Engineering, The City College of New York, New York, New York 10031
}

Handling $(\mathrm{H})$ and cross-fostering $(\mathrm{CF})$ rodent pups during postnatal development triggers changes in maternal behavior which in turn trigger long-term physiological changes in the offspring. However, less is known about the short-term effects of $\mathrm{H}$ and $\mathrm{CF}$ on infant development. In this study we hypothesized that manipulations of maternal care affect the onset of hearing in Wistar rats. To test this hypothesis we obtained auditory brainstem responses (ABRs) and micro-CT $\mathrm{x}$-ray scans to measure changes in the development of the auditory periphery in $\mathrm{H}$ and $\mathrm{CF}$ pups manipulated at postnatal day (P)1, P5, or P9. We found evidence of changes in hearing development in $\mathrm{H}$ and CF pups compared with naive pups, including changes in the percentage of animals with ABRs during development, a decrease in ABR thresholds between P13 and P15, and anatomical results consistent with an accelerated formation of the middle ear cavity and opening of the ear canal. Biochemical measurements showed elevated levels of thyroid hormone in plasma from naive and CF pups. These results provide evidence that manipulations of maternal care accelerate hearing onset in Wistar rats. Understanding the mechanisms by which maternal care affects hearing onset opens new opportunities to study experiencedependent development of mammalian hearing.

Key words: auditory development; enrichment; experience-dependent development; middle-ear development; plasticity; thyroid hormone

\section{Introduction}

The onset of auditory function in humans takes place in utero (Ruben, 1992). In contrast, hearing development in altricial species, such as rats, occurs after birth (Rubel, 1984). Such differences indicate that genetic mechanisms are involved in initiating hearing function in different mammalian species. Whether experience also plays a role in timing the onset of mammalian hearing remains unclear.

Previous studies used altricial rodents to study how maternal care affects infant development. Licking and grooming (LG) and arched-back nursing $(\mathrm{ABN})$ are maternal care behaviors displayed by female rats and mice. Manipulations of maternal care studies showed that when pups are handled $(\mathrm{H})$, this changes the mother's behavior, increasing LG (Liu et al., 1997). Furthermore, in cross-fostering (CF) experiments, in which adoptive mothers

\footnotetext{
Received Sept. 29, 2013; revised Jan. 31, 2014; accepted Feb. 19, 2014.

Author contributions: S.A., A.S., N.M., V.K., L.C., and A.R.-C. designed research; S.A., A.S., N.M., V.K., L.C., and A.R.-C. performed research; S.A., A.S., N.M., V.K., L.C., and A.R.-C. contributed unpublished reagents/analytic tools; S.A., A.S., N.M., V.K., L.C., and A.R.-C. analyzed data; S.A., A.S., N.M., V.K., L.C., and A.R.-C. wrote the paper.

This work was supported by Research Enhancement Award 5SC1HD068129 from the Eunice Kennedy Shriver National Institute of Child Health and Human Development (A.R.-C.), and grants from the National Center for Research Resources (2G12RR03060-26A1) and the National Institute on Minority Health Disparities (8G12MD007603-27) from the National Institutes of Health. We thank M. Melecio-Vazquez for technical assistance, Dr K. Ali for kindly providing access to the plate spectrophotometer used in ELISA experiments, and Drs 0. Tchernichovski and J.L. Peña for their comments on a previous version of the paper.

The authors declare no competing financial interests.

Correspondence should be addressed to Dr Adrián Rodríguez-Contreras, Biology Department, The City College of New York, 160 Convent Avenue, Marshak Hall, Room MR526, New York, NY 10031. E-mail: arodrig@sci.ccny.cuny.edu.

DOI:10.1523/JNEUROSCI.4188-13.2014

Copyright $\odot 2014$ the authors $\quad 0270-6474 / 14 / 344528-06 \$ 15.00 / 0$
}

raise pups, it was found that rodent dams have increased contact time with pups, increased LG-ABN behaviors, and a decreased retrieval response latency (Maccari et al., 1995). Pups reared by high LG-ABN mothers have increased spatial learning and memory compared with pups of low LG-ABN mothers (Caldji et al., 1998). A reduced stress response and decreased fearfulness has been reported in the case of $\mathrm{H}$ pups (Caldji et al., 1998). In addition, Guzzetta et al. (2009) hypothesized that acceleration of brain development in enriched environments is related to an increased LG behavior in mother rats. To test this idea, they showed that massaging pups before eye opening (to mimic increased LG) accelerated the development of visually evoked potentials (Guzzetta et al., 2009). It is not known whether changes in maternal care affect auditory development.

Because rats are naturally born with fused ear canals and mesenchyme in the middle ear (Saunders et al., 1993; Mulder et al., 1998), we reasoned that analysis of the functional and anatomical changes in the auditory periphery during development should provide insight on whether changes in experience affect the onset of hearing in altricial rodents. We used $\mathrm{H}$ and $\mathrm{CF}$ to test the hypothesis that maternal care affects hearing onset in Wistar rats (Fig. 1).

\section{Materials and Methods}

Animals. Experimental procedures were reviewed and approved by the Institutional Animal Care and Use Committee of the City College of New York. Timed-pregnant first time Wistar dams were obtained at E15 (Charles River Laboratories; RRID: RGD:737929). The day of birth was considered postnatal day $(\mathrm{P}) 0$. Pups of either sex were used in experiments. Animals were housed in $38 \times 16 \times 31 \mathrm{~cm}$ Plexiglas cages. Room 
A

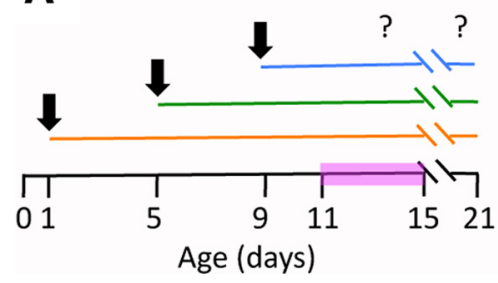

B

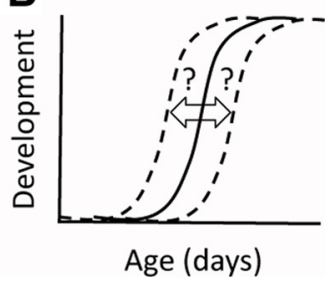

Figure 1. Experiment design and hypothesis. $A$, Rat pups were $\mathrm{CF}$ or $\mathrm{H}$ at $\mathrm{P} 1, \mathrm{P} 5$, or $\mathrm{P9}$ (indicated with black arrows). Developmental changes in the auditory periphery were analyzed during the onset of hearing (pink bar) and at P21. B, Expected effects of manipulating maternal care on hearing development. The hypothesis predicts developmental changes in $\mathrm{H}$ or $\mathrm{CF}$ pups (dashed lines) compared with naives (solid line). A shift to the left would indicate accelerated development. A shift to the right would indicate a developmental delay. The null hypothesis is no change compared with naive pups ( $\boldsymbol{B}$, solid line).

temperature $\sim 24^{\circ} \mathrm{C}$, humidity $\sim 40 \%$, bedding change twice a week, $12 \mathrm{~h}$ light/dark cycle (lights on at 6:00 A.M.) and ad libitum access to food (5053-PicoLab Rodent Diet 20, LabDiet) and water were provided.

Manipulations of maternal care. We chose to study the effects of $\mathrm{H}$ and $\mathrm{CF}$ )at P1, P5, or P9 (Fig. 1A). We refer to these animals as HP1, HP5, HP9, CFP1, CFP5, and CFP9 pups. H or CF pups were taken from their cages, weighed, and then placed back in a cage with their biological dam or a foster one, respectively (handling duration 10-15 min during the light phase, between 1:00 and 5:00 P.M.). Dams that gave birth within $6 \mathrm{~h}$ of each other were included in CF experiments. During weighing and handling pups were covered in bedding from the foster cage to mask their smell. Except for bedding change twice a week naive, H, and CF litters were left undisturbed until testing day.

ABRs. We measured auditory brainstem responses (ABRs) in 319 pups. Different groups of naive, $\mathrm{H}$, and CF pups were tested at every age without repeated measures on any animal. Naive pups were tested at P11, $\mathrm{P} 12$ ( $n=8$ each), P13, P14 ( $n=10$ each), P15 $(n=14)$, and P21 $(n=19)$. CFP1 pups were tested at P11 $(n=4), \mathrm{P} 12, \mathrm{P} 13, \mathrm{P} 14, \mathrm{P} 15$ ( $n=8$ each), and P21 $(n=9)$. CFP5 pups were tested at P11, P12 ( $n=8$ each), P13, $\mathrm{P} 14(n=12$ each), P15 $(n=14)$, and P21 $(n=11)$. CFP9 pups were tested at P11, P12 $(n=4$ each), P13 $(n=9), \mathrm{P} 14, \mathrm{P} 15$, and $\mathrm{P} 21(n=10$ each). HP1 pups were tested at P11, P12 $(n=7$ each), P13 $(n=7), \mathrm{P} 14$ $(n=6), \mathrm{P} 15$, and P21 $(n=5$ each). HP5 pups were tested at P11, P12 ( $n=5$ each), P13 $(n=6), \mathrm{P} 14(n=8), \mathrm{P} 15(n=7)$, and P21 $(n=4)$. HP9 pups were tested at P11, P12, P13, P14, P15 ( $n=3$ each), and P21 $(n=6)$.

Briefly, rats were anesthetized with a mixture of medical oxygen and $3 \%$ isoflurane, followed by intraperitoneal injections of ketamine (41.7 $\mathrm{mg} / \mathrm{kg})$ and xylazine $(2.3 \mathrm{mg} / \mathrm{kg})$. Anesthetized pups were placed in the prone position on a heating pad set to $37^{\circ} \mathrm{C}$, inside a double wall soundattenuating chamber (IAC). ABRs were obtained using subdermal electrodes (GRASS RA4LI), as described by Ping et al. (2007), or as described by Bogaerts et al. (2009). Auditory stimuli consisted of $20 \mu$ s long broadband clicks $(1-50 \mathrm{kHz})$ with intensities varying from 82 to $2 \mathrm{~dB}$ sound pressure level, in $5 \mathrm{~dB}$ decrements through a free-field Kanetec MB-FX speaker placed $34 \mathrm{~mm}$ horizontally from the right ear. Free-field auditory stimuli were generated with TDT System 3 hardware (Tucker-Davis Technologies) at $20 \mathrm{kHz}$ and presented at a rate of $40 \mathrm{~Hz}$. Click polarity was alternated to minimize the presence of stimulus artifacts. Stimuli were calibrated with a type $7012 \frac{1}{2} 2$ inch ACO Pacific microphone. ABRs were acquired with a Medusa preamplifier, saved to hard disk, and inspected at the end of every sound presentation. ABR waveforms were analyzed more carefully offline using Igor Pro (Wavemetrics).

Threshold and wave 1 measurements. Thresholds were defined by the lowest click intensity that produced a visually detectable ABR wave 1 (Ping et al., 2007). Pups with a single ABR wave 1 at $82 \mathrm{~dB}$, the highest click intensity used in this study, were considered hearing animals with high ABR thresholds and were counted in the percentage of pups with an ABR (Fig. 2). Wave 1 latency was measured at $82 \mathrm{~dB}$ in naive, $\mathrm{H}$, and $\mathrm{CF}$ pups at P15 and P21.

Hormone measurements. In separate experiments, we obtained serum from 12 naive and 12 CFP5 pups for hormone control experiments.
Animals were killed rapidly by decapitation between 9:00 and 11:00 A.M. at $\mathrm{P} 6, \mathrm{P} 9, \mathrm{P} 12$, and $\mathrm{P} 15$ ( $n=3$ pups per age). Blood samples were collected in $1 \mathrm{ml}$ Eppendorf tubes, left at room temperature for $20 \mathrm{~min}$, and centrifuged at $4000 \times g$ for $15 \mathrm{~min}$ (Microcentrifuge 5424R, Eppendorf). The supernatant was stored in $300 \mu \mathrm{l}$ aliquots at $-20^{\circ} \mathrm{C}$ until used. Plasma Corticosterone (CORT; Enzo Life Sciences, catalog \#EK377, RRID: RRID:AB_2307314), Insulin-like growth factor 1 (IGF-1; Boster Biological Technology, catalog \#ADI-900-097, RRID:AB_2307315), Leptin (Millipore, catalog \#EZRL-83K, RRID:AB_2307316), and Tri-iodothyronine (T3, CUSABIO, catalog \#CSB-E05085r, RRID: AB_2307317) were measured by ELISA using a multiplate reader (SpectraMax5, Molecular Devices) according to manufacturer's instructions. Due to insufficient amount of serum sample IGF-1 could not be measured in two naive and three CFP5 pups at P6.

High-resolution $x$-ray tomography, image processing, and $3 D$ measurements. In separate experiments, microcomputer tomography (microCT) $\mathrm{x}$-ray scans were conducted in 60 animals. All the samples were scanned immediately after decapitation. Images were acquired using a desktop micro-CT system equipped with10 MP digital detector, 10W power energy (100 KV and $100 \mathrm{~mA}$ ), and a $0.5 \mathrm{~mm}$ aluminum filter (1172, Bruker SkyScan). X-Ray projections were generated around the samples using $0.4^{\circ}$ rotation steps with a resolution of $11.5 \mu \mathrm{m}$ per pixel (five exposures per projection).

A modified back-projection reconstruction algorithm (NRecon, v1.6.1.2; Feldkamp et al., 1984) was used to generate cross-section images from the planar x-ray projections. All reconstruction parameters were applied identically to all scans, with the exception of the postalignment compensation, which does not compromise comparative measurements (Gu et al., 2012; Souzanchi et al., 2012).

Scans were loaded into MIMICS (v14.0, Materialise) for segmentation and $3 \mathrm{D}$ reconstruction. Skull, soft tissues, and air were identified and segmented using global thresholds and 3D region growing algorithms (Palacio-Mancheno et al., 2014). The volume of air present in each ear canal was measured directly from the segmented images. A 3D reconstruction of the bone was performed to measure the middle ear cavity (bulla) and cranium dimensions. Minimal diameter of the ear canal was determined by identifying the narrowest section of the conduct, as well as its surface area at the location of minimal diameter.

Analysis and statistics. Unless indicated, data represent mean $\pm \mathrm{SD}$. Curve fitting and statistical analysis were done with Prism 6 (GraphPad Software). Goodness of fit was evaluated with the least-squares method. ABR thresholds and micro-CT $\mathrm{x}$-ray scan datasets were tested for normality using the D'Agostino and Pearson omnibus K2 test (D'Agostino, 1986). Functional and anatomical changes during postnatal age were assessed with a two-way ANOVA Dunnett's multiple-comparisons test. Serum hormone levels were analyzed with two-way ANOVA Sidak's multiple-comparisons test. $A_{50}$ values and $A B R$ threshold rates (see below) were compared with the extra sum of squares $F$ test. Significance criterion was $p<0.05$.

Equation 1 was used to fit data in Figures 2 and 4:

$$
Y=Y_{0}+\left(Y_{\max }-Y_{0}\right) /\left[1+\exp \left(A_{50}-X / k\right)\right] .
$$

Where $\mathrm{Y}_{0}$ is the minimum observed $Y$ (i.e., percentage of pups with $A B R$, air volume, or ear canal diameter), $Y_{\max }$ is the maximum observed $Y, A_{50}$ is the age at which $Y$ is half maximum, $X$ is age (in days), and $k$ is the rate constant.

Data in Figure 2D,E was fitted to Equation 2:

$$
Y=\left(Y_{0}-\text { plateau }\right) \exp [-(k \mathrm{X})+\text { plateau }],
$$

where $\mathrm{Y}_{0}$ is the $\mathrm{ABR}$ threshold at $\mathrm{P} 0$, plateau is the ABR threshold at $\mathrm{P} 21$, $k$ is the rate constant, and $X$ is age (in days).

\section{Results}

\section{Accelerated hearing onset in CFP5 and $\mathrm{H}$ pups: percentage of pups with ABRs}

We found that the percentage of naive pups with ABRs gradually increased from zero at P11 and $\mathrm{P} 12$ to $40 \%$ at $\mathrm{P} 13,80 \%$ at $\mathrm{P} 14$, 
$92 \%$ at $\mathrm{P} 15$, and $100 \%$ at $\mathrm{P} 21$ (Fig. $2 \mathrm{~A}$ ). In comparison, at P13 CFP1 and CFP9 groups showed $38 \%$ and $44 \%$ of pups with ABRs, respectively, whereas $100 \%$ of pups showed ABRs in the CFP5 group (Fig. $2 A)$. Although $25 \%$ of pups at P12 had ABRs in the CFP1 group, we did not note major differences between CFP1 and naive pups at subsequent ages. The percentage of CFP9 pups with ABRs was almost identical to the naive group profile. To obtain a more quantitative assessment of these observations, we used Equation 1 to fit data in Figure 2 and obtained $A_{50}$ values that we compared between naive and $\mathrm{CF}$ or $\mathrm{H}$ pups. We calculated an $A_{50}$ value of $13.27 \pm 0.08 \mathrm{~d}$ in naive pups and found a statistically significant difference with the $A_{50}$ value calculated for CFP5 pups, but not with $A_{50}$ values obtained for CFP1 or CFP9 pups (extra sum of squares $F$ test; CFP1: $A_{50}=13.04 \pm 0.18 \mathrm{~d}, p=0.2345$; CFP5: $A_{50}=12.50 \pm 0.18 \mathrm{~d}, p=0.0048$; and CFP9: $A_{50}=13.22 \pm 0.11 \mathrm{~d}, p=$ 0.5218; Fig. 2C).

We found that $\mathrm{H}$ groups had a higher percentage of animals with ABRs compared with naive pups (Fig. $2 B$ ). Fitting data in Figure $2 B$ to Equation 1 gave $A_{50}$ values of $12.72 \pm 0.03 \mathrm{~d}$ in HP1 pups, $12.89 \pm 0.04 \mathrm{~d}$ in HP5 pups, and $12.81 \pm$ $0.05 \mathrm{~d}$ in HP9 pups, all of which were significantly different from the naive $A_{50}$ value (extra sum of squares $F$ test; HP1, $p<0.0001$; HP5, $p=0.0003$; HP9, $p=0.0004$; Fig. $2 C)$. Overall, these results suggested an accelerated development of $\sim 0.2 \mathrm{~d}$ in $\mathrm{H}$ pups and $0.5 \mathrm{~d}$ in CFP5 pups compared with naive animals.

\section{Lower ABR thresholds in CF pups during hearing onset but not at P21}

As shown in Figure 2D, ABR thresholds in naive, CFP1, and CFP5 animals decreased between P13 and P21 (two-way ANOVA, Dunnett's multiple-comparison test; age: $F=58.67, p<0.0001$; maternal care condition: $F=10.07, p<0.0001)$. Comparison of ABR thresholds between naive pups and CF animals showed significant differences at P13, and P15 in CFP1 pups; at P14 in CFP5 pups; and at P13 and P14 in CFP9 pups (orange, green, and blue asterisks in Fig. 2D, respectively). We did not find statistically significant differences between naive and CF pups at P21. These results suggested that $A B R$ thresholds could have a faster rate of change in CF pups compared with naives. To check this interpretation, we fitted data in Figure $2 D$ to Equation 2 and obtained estimates of the rate of ABR threshold change during postnatal development (Fig. 2D, color lines). However we did not find any statistically significant difference between naive and CF rates (Fig. $2 F$; extra sum of squares $F$ test; CFP1, $p=0.4326$; CFP5, $p=$ 0.0984; CFP9, $p=0.3387$ ).

We performed a similar analysis of ABR thresholds between naive and $\mathrm{H}$ pups. As shown in Figure $2 E$, ABR thresholds decreased between P13 and P21 (two-way ANOVA, Dunnett's multiple-comparison test; age, $F=62.49, p<0.0001$; maternal care condition, $F=2.95, p=0.0369$ ). We did not find statistically significant differences between naive and $\mathrm{H}$ pup ABR thresholds at any age. Fitting data in Figure $2 E$ to Equation 2, we obtained in C represent SEM.
B

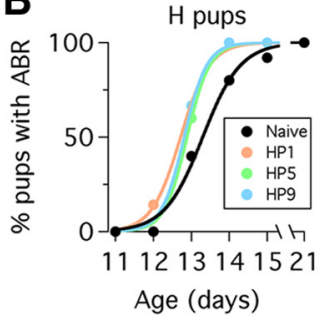

E

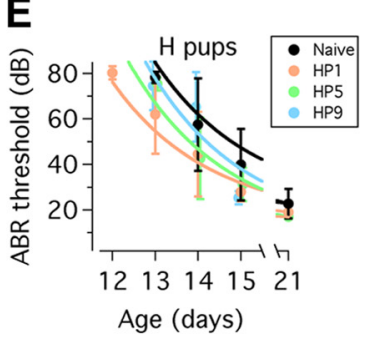

C

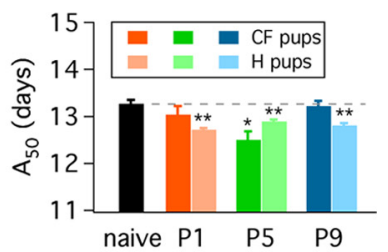

$\mathbf{F}$

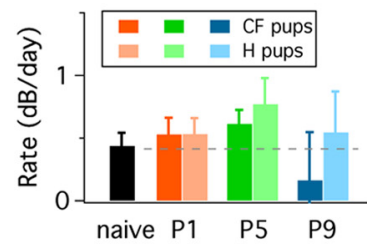

Figure 2. Functional changes during the onset of hearing in naive, $\mathrm{CF}$ and $\mathrm{H}$ groups. $A$, Percentage of pups with $\mathrm{ABRs}$ in $\mathrm{CF}$ (itained from fits to equation 1. Color indicates the age of manipulation: P1 (orange), P5 (green), and P9 (blue). Bright colors different postnatal ages. Black circles represent naive pup data in $\boldsymbol{A}$ and $\boldsymbol{B}$. Asterisks indicate statistically significant differe

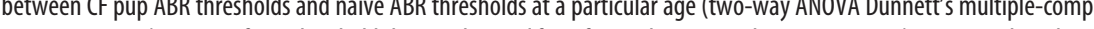
Dashed line represents naive $A B R$ threshold rate of change, also shown as a black bar. $A, B$, Data represent mean $\pm S D$. Error bars

A

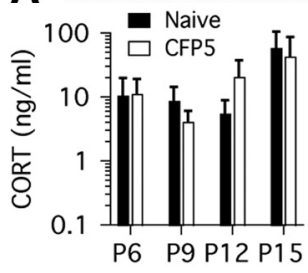

C

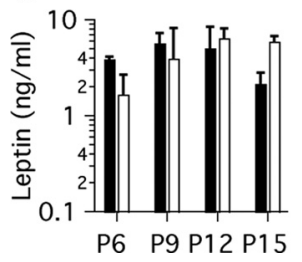

B

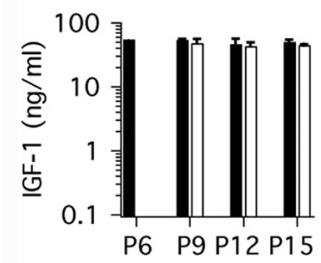

D

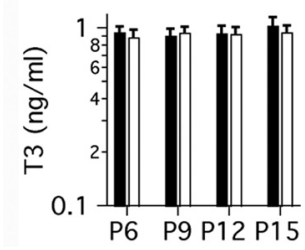

Figure 3. Plasma hormone levels in naive and CFP5 pups. Serum samples were obtained and analyzed from three pups at $\mathrm{P} 6, \mathrm{P} 9, \mathrm{P} 12$ and $\mathrm{P} 15 . \mathrm{A}$, CORT concentration is low in $\mathrm{P} 6-\mathrm{P} 12$ pups, and increases in $P 15$ pups. B, IGF-1 concentration did not change between $P 9$ and P15. Note that only one pup sample was measured at P6 due to limitations in available serum at that age. Leptin $(\boldsymbol{C})$ and T3 (D)concentrations did not change significantly between P6 and P15. Data represent mean and SD. Bar labels in $A$ apply to all panels.

estimates of the rate of ABR threshold change (Fig. 2E, color lines). However, we did not find significant statistical differences between the rates obtained for $\mathrm{H}$ pup data compared with the naive pup rate (Fig. 2F; HP1, $p=0.4663$; HP5, $p=0.0794$; HP9, $p>0.5)$.

We examined the latency of wave 1 at P15 and P21, and did not find statistically significant differences between naive, $\mathrm{H}$ and CF groups (data not shown). Overall, these results suggested that ABR thresholds were lower in some CF pups during the onset of 

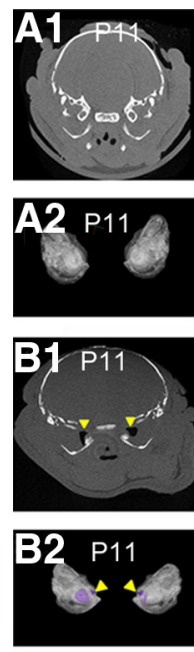
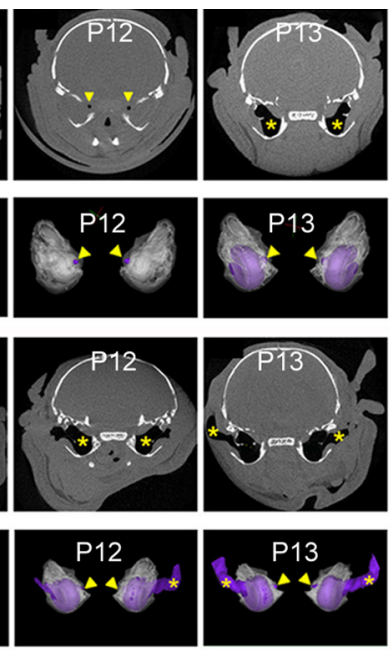
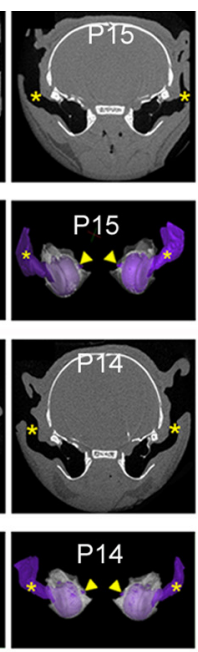
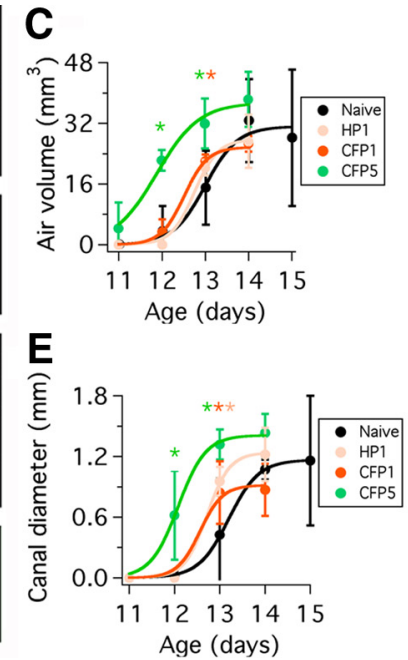

D

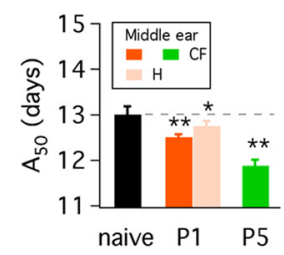

$\mathbf{F}$

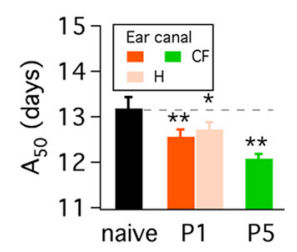

Figure 4. Anatomical changes in naive, HP1, CFP1, and CFP5 pups. A1, Micro-CT x-ray scans of naive pups from ages P11-P15. Gray represents soft tissue, white represents bone, and black represents air in the middle ear and auditory canal (indicated by yellow arrowheads and asterisks). A2, 3D reconstructions of left and right ear scans of animals shown in $\boldsymbol{A}$ 1. The presence of small air pockets (purple) next to the medial wall of the middle ear cavity is noticeable at P12 (yellow arrowheads). At P13, middle-ear cavitation is complete, and at P15 air is observed in the ear canal (yellow asterisks). B1, Micro-CT x-ray scans of (FP5 pups from ages P11-P14. B2, 3D reconstructions of scan data shown in B1. The presence of small air pockets (purple) next to the medial wall of the middle ear cavity is noticeable at P11 (yellow arrowheads). At P12, middle-ear cavitation is complete, and at P14 air is observed in the ear canal (yellow asterisks). $\boldsymbol{C}$, Measurements of air volume in the middle ear obtained from the micro-CT x-ray scans such as those shown in $\boldsymbol{A}$ and $\boldsymbol{B}$. $\boldsymbol{D}$, Summary of $A_{50}$ values obtained from fitting data in $\boldsymbol{C}$ to Equation 1 (see Materials and Methods). $\boldsymbol{E}$, Micro-CT x-ray scan data measurements of auditory canal diameter obtained from micro-CT x-ray scans such as those shown in $\boldsymbol{A}$ and $\boldsymbol{B}$. $\boldsymbol{F}$, Summary of $A_{50}$ values obtained from fitting data in $\boldsymbol{E}$ to Equation 1 (see Materials and Methods). Dashed line represents naive $A_{50}$ value in $\boldsymbol{D}$ and $\boldsymbol{F}$, which is also shown as a black bar. Asterisks indicate statistical differences compared with naive data ${ }^{*} p<$ $\left.0.01 ;{ }^{* *} p<0.001\right)$. Error bars in $\boldsymbol{C}$ and $\boldsymbol{E}$ represent SD; error bars in $\boldsymbol{D}$ and $\boldsymbol{F}$ represent SEM.

hearing, but that any differences observed were no longer present at P21.

\section{Plasma hormone levels in naive and CFP5 pups}

To determine whether a modulation of hormone levels could be involved in the effects of maternal care on auditory development, we measured serum levels of four different hormones in naive and CFP5 pups at different ages (Fig. 3). We obtained similar plasma CORT levels in naive and CFP5 pups between P6 and P12, and found a trend to increased levels at P15. We did not find differences between CORT levels in naive and CFP5 pups (Fig. $3 A$; two-way ANOVA; maternal care condition, $F=0.0141, p=$ 0.9071 ; age, $F=4.352, p=0.0201)$. We did not find differences in the IGF-1 plasma levels between P9 and P15 in naive and CFP5 pups (Fig. 3B; two-way ANOVA; maternal care condition: $F=$ 2.655, $p=0.1292$; age: $F=1.555, p=0.2510)$. We found that Leptin levels showed a trend to increase between P6 and P12, followed by decrease at P15. However, these changes were not significant (Fig. 3C; two-way ANOVA; maternal care condition: $F=0.1106, p=0.7437$; age: $F=2.012, p=0.1529)$. Last, we found that T3 levels did not change between P6 and P15, and there were no differences between naive and CFP5 pups (Fig. 3D; two-way ANOVA; maternal care condition: $F=0.5807, p=$ 0.4571 ; age: $F=7691, p=0.5280$ ). These results provide information on the plasma hormone profile of rat pups in this study.

Anatomical changes in middle- and external-ear development in naive and manipulated pups

Based on the above results, we chose to perform separate micro-CT x-ray scanning experiments to examine the development of the middle ear and auditory canal in naive, HP1, CFP1, and CFP5 pups (Fig. 4). Middle-ear cavitation in naive pups started as tiny air pockets that increased in size in older animals. Air pockets increased in size from the medial wall of the middle ear at P12 toward the tympanic membrane at P13. By P15, a fully formed middle ear cavity and ear canal were present (Fig. 4A1). Similar age-dependent changes were observed in HP1 and CFP1 pups, with the exception of CFP5 pups, in which air pockets occurred as early as P11 (Fig. 4B1). 3D reconstructions of the micro-CT x-ray scan data corroborated that air filled the middle ear cavity before the auditory canal was fully formed in naive and CF pups. This approach also demonstrated the earlier development of the middle ear cavity and auditory canal in CFP5 pups compared with naive pups (Fig. 4A2,B2; see 3D reconstructions).

We also found a gradual increase in middle ear air volume with age in naive (Fig. $4 C$; one-way ANOVA, $F=10.73, p<$ 0.0001), HP1 $(F=58.98, p<0.0001)$, CFP1 $(F=296.3, p<$ $0.0001)$, and CFP5 pups $(F=46.93, p<0.0001)$. Furthermore, we found significant differences in middle ear air volume of CFP5 pups with respect to naive pups at ages $\mathrm{P} 12$ and $\mathrm{P} 13$, and at age P13 in CFP1 pups compared with naive pups (Fig. 4C, color asterisks; Dunnett's multiple-comparisons test). Next, we fitted data in Figure $4 C$ to Equation 1 and obtained an $A_{50}$ value of $13 \pm$ $0.18 \mathrm{~d}$ in naive animals. We found statistically significant differences between the naive $A_{50}$ value and $A_{50}$ values for HP1 pups $(12.75 \pm 0.11 \mathrm{~d} ; p=0.0133)$, CFP1 pups $(12.51 \pm 0.06 \mathrm{~d} ; p<$ $0.0001)$, and CFP5 pups $(11.88 \pm 0.13 \mathrm{~d} ; p=0.0032)$.

The results described above showed quantitative differences in middle-ear cavitation between naive and manipulated pups. Next, we measured ear canal diameter during development. We found that there were significant differences in ear canal diameter with respect to naive pups at $\mathrm{P} 13$ in $\mathrm{HP} 1$ and CFP1 pups, and at ages P12 and P13 in CFP5 pups (Fig. 4E, color asterisks; Dunnett's multiple-comparisons test). We fitted data in Figure $4 E$ to Equation 1 and obtained an $A_{50}$ value of $13.18 \pm 0.25 \mathrm{~d}$ in naive pups. We found statistically significant differences between the naive $A_{50}$ value and $A_{50}$ values for HP1 pups $(12.72 \pm 0.17 \mathrm{~d} ; p=$ $0.0077)$, CFP1 pups (12.56 $\pm 0.16 \mathrm{~d} ; p<0.0001)$, and CFP5 pups $(12.08 \pm 0.10 \mathrm{~d} ; p<0.0001)$. Altogether, these results showed 
evidence of accelerated middle-ear cavitation and ear-canal formation in $\mathrm{H}$ and CF pups.

\section{Discussion}

The onset of hearing in mammals is characterized by the formation of an air-filled middle ear cavity and clearance of the auditory canal, in conjunction with rapid changes in auditory thresholds and behavioral responses to sound (Rubel, 1984). The mechanisms that control such coordinated developmental changes are not entirely understood, although previous and recent studies indicate that endocrine and autocrine signals are involved (for review, see Ng et al., 2013). To our knowledge, the results of this study showed for the first time that $\mathrm{H}$ and $\mathrm{CF}$ manipulations in Wistar rats trigger physiological and anatomical changes in the auditory periphery that are consistent with an acceleration of hearing onset (Fig. 1). Furthermore, our study demonstrates for the first time that middle ear cavitation precedes ear canal opening in an inside-out formation pattern (Fig. $4 A 2, B 2)$.

Analysis of the percentage of pups with ABRs showed differences between naive and manipulated pups. For example, using ABRs we estimated that hearing onset was accelerated by $\sim 0.2$ and $0.5 \mathrm{~d}$ in $\mathrm{H}$ and CFP5 pups, respectively (Fig. 2). It is intriguing that only $\mathrm{H}$ pups showed evidence of accelerated development when manipulated at P1 and P9, whereas both $\mathrm{H}$ and CF pups showed evidence of accelerated development when manipulated at P5 (Fig. 2C). Although we found some significant differences in ABR thresholds between naive and CF pups (Fig. 2D), these effects did not translate to differences in the rate of ABR threshold change during development. Note that we did not detect statistically significant differences in $\mathrm{ABR}$ thresholds between naive and $\mathrm{H}$ pups. This suggests that ABRs might not be a very sensitive tool to detect functional differences in developing animals. In the case of CF pups however, ABR threshold differences can be detected only during the few days spanning the onset of hearing. Naive and manipulated animals achieve a similar maturation state very rapidly, because no differences were detected at P21.

Opening of the ear canal is considered a limiting step for hearing development in altricial mammals (Rubel, 1984; Moore and Hine, 1992; Geal-Dor et al., 1993). Therefore, the results described in Figure 2 predicted that HP1, CFP1, and CFP5 pups should have noticeable changes in the structure of the auditory periphery during development. We found support for this prediction (Fig. 4). Interesting to us, middle-ear cavitation and opening of the ear canal occurred almost a full day ahead in CFP5 pups compared with naive, HP1, and CFP1 pups. These functional and anatomical observations are consistent with the proposal that the first 2 weeks of postnatal life constitute a sensitive period in auditory development (Knipper et al., 2000). We think this interpretation is further supported by our hormone measurements (Fig. 3).

First, CORT levels in naive and CFP5 pups were consistent with previous reports (Guzzetta et al., 2009; Oomen et al., 2009), suggesting that our results did not involve changes in pups' stress levels. Second, although T3 hormone levels were not different between naive and CFP5 pups, our measurements at P6 showed higher baseline levels with respect to data from previous studies (Knipper et al., 1998). Because T3 deficiency is known to cause irreversible damage to the auditory system during the sensitive period outlined above (Knipper et al., 2000), it is possible that higher baseline T3 plasma levels are important for the effects of $\mathrm{H}$ and $\mathrm{CF}$ on auditory development described in this study. We also found similar plasma Leptin levels to those reported previously
(Smith and Waddell, 2003). Although we did not find major differences in plasma hormone concentrations in any of the hormones measured in this study, we cannot rule out the participation of autocrine and paracrine signaling, or changes in receptor expression in target tissues. Indeed, an increase in tissue-specific IGF-1 is involved in enrichment effects on visual cortical development and is important for normal cochlear development (for IGF-1, see Ciucci et al., 2007; Guzzetta et al., 2009; SanchezCalderon et al., 2010; for T3, see Ng et al., 2013). Similarly, changes in receptor expression during development have been described for Leptin (Smith and Waddell, 2003).

A pitfall of our study is that we did not monitor maternal care. This is important because previous studies identified a significant interaction between maternal care and housing conditions, particularly in offspring of low LG-ABN mothers (Francis et al., 2002; Bredy et al., 2003; Champagne and Meaney, 2007). Given the variability of maternal care behavior in rodents (Jensen Peña and Champagne, 2013), it is surprising that we detected significant functional and structural differences in $\mathrm{H}$ and $\mathrm{CF}$ pups. We suspect that the magnitude of functional and anatomical changes we observed could be related to natural variations in maternal behavior. In future studies, tracking maternal care could help control for important behavioral differences in mothers and provide a framework to evaluate the variability of pup's measurements. The results of this study should stimulate further research concerning identification of the sensory cues that trigger behavioral changes in lactating dams, and the signaling pathways that are activated by such cues to change maternal behavior (Champagne et al., 2001). Last, we propose that our results also have important implications for understanding how manipulations of maternal care, housing, and rearing conditions affect developmental responses to sensory stimulation in the peripheral and central auditory system of mammalian infants (Froemke and Jones, 2011).

\section{References}

Bogaerts S, Clements JD, Sullivan JM, Oleskevich S (2009) Automated threshold detection for auditory brainstem responses: comparison with visual estimation in a stem cell transplantation study. BMC Neurosci 10:104-110. CrossRef Medline

Bredy TW, Humpartzoomian RA, Cain DP, Meaney MJ (2003) Partial reversal of the effect of maternal care on cognitive function through environmental enrichment. Neuroscience 118:571-576. CrossRef Medline

Caldji C, Tannenbaum B, Sharma S, Francis D, Plotsky PM, Meaney MJ (1998) Maternal care during infancy regulates the development of neural systems mediating the expression of fearfulness in the rat. Proc Natl Acad Sci U S A 95:5335-5340. CrossRef Medline

Champagne FA, Meaney MJ (2007) Transgenerational effects of social environment on variations in maternal care and behavioral response to novelty. Behav Neurosci 121:1353-1363. CrossRef Medline

Champagne F, Diorio J, Sharma S, Meaney MJ (2001) Naturally occurring variations in maternal behavior in the rat are associated with differences in estrogen-induced central oxytocin receptors. Proc Natl Acad Sci U S A 98:12736-12741. CrossRef Medline

Ciucci F, Putignano E, Baroncelli L, Landi S, Berardi N, Maffei L (2007) Insulin-like growth factor 1 (IGF-1) mediates the effects of enriched environment (EE) on visual cortical development. PLoS ONE 2:e475. CrossRef Medline

D’Agostino RB (1986) Tests for the normal distribution. In: Goodness-offit techniques (D’Agostino RB, Stephens MA, eds), pp 367-413. New York: Marcel Dekker.

Feldkamp LA, Davis LC, Kress JW (1984) Practical cone-beam algorithm. J Opt Soc Am 1:612-619. CrossRef

Francis DD, Diorio J, Plotsky PM, Meaney MJ (2002) Environmental enrichment reverses the effects of maternal separation on stress reactivity. J Neurosci 22:7840-7843. Medline 
Froemke RC, Jones BJ (2011) Development of auditory cortical synaptic receptive fields. Neurosci Biobehav Rev 35:2105-2113. CrossRef Medline

Geal-Dor M, Freeman S, Li G, Sohmer H (1993) Development of hearing in neonatal rats: air and bone conducted ABR thresholds. Hear Res 69:236242. CrossRef Medline

Gu XI, Palacio-Mancheno PE, Leong DJ, Borisov YA, Williams E, Maldonado N, Li YH, Laudier D, Majeska RJ, Schaffler MB, Sun HB, Cardoso L (2012) High resolution micro arthrography of hard and soft tissues in a murine model. Osteoarth Cartil 20:1011-1019. CrossRef Medline

Guzzetta A, Baldini S, Bancale A, Baroncelli L, Ciucci F, Ghirri P, Putignano E, Sale A, Viegi A, Berardi N, Boldrini A, Cioni G, Maffei L (2009) Massage accelerates brain development and the maturation of visual function. J Neurosci 29:6042-6051. CrossRef Medline

Jensen Peña C, Champagne FA (2013) Implications of temporal variation in maternal care for the prediction of neurobiological and behavioral outcomes in offspring. Behav Neurosci 127:33-46. CrossRef Medline

Knipper M, Nandtlow C, Gestwa L, Köpschall I, Rohbock K, Wiechers B, Zenner HP, Zimmermann U (1998) Thyroid hormone affects Schwann cell and oligodendrocyte gene expression at the glial transition zone of the VIIIth nerve prior to cochlea function. Development 125:3709-3718. Medline

Knipper M, Zinn C, Maier H, Praetorius M, Rohbock K, Köpschall I, Zimmermann U (2000) Thyroid hormone deficiency before the onset of hearing causes irreversible damage to peripheral and central auditory systems. J Neurophysiol 83:3101-3112. Medline

Liu D, Diorio J, Tannenbaum B, Caldji C, Francis D, Freedman A, Sharma S, Pearson D, Plotsky PM, Meaney MJ (1997) Maternal care, hippocampal glucocorticoid receptors, and hypothalamic-pituitary-adrenal responses to stress. Science 277:1659-1662. CrossRef Medline

Maccari S, Piazza PV, Kabbaj M, Barbazanges A, Simon H, Le Moal M (1995) Adoption reverses the long-term impairment in glucocorticoid feedback induced by prenatal stress. J Neurosci 15:110-116. Medline

Moore DR, Hine JE (1992) Rapid development of the auditory brainstem response threshold in individual ferrets. Brain Res Dev Brain Res 66:229235. CrossRef Medline

Mulder JJ, Kuijpers W, Peters TA, Tonnaer EL, Ramaekers FC (1998) De- velopment of the tubotympanum in the rat. Laryngoscope 108:18461852. CrossRef Medline

Ng L, Kelley MW, Forrest D (2013) Making sense with thyroid hormone: the role of T3 in auditory development. Nat Rev Endocrinol 9:296-307. CrossRef Medline

Oomen CA, Girardi CE, Cahyadi R, Verbeek EC, Krugers H, Joëls M, Lucassen PJ (2009) Opposite effects of early maternal deprivation on neurogenesis in male versus female rats. PLoS ONE 4:e3675. CrossRef Medline

Palacio-Mancheno PE, Larriera AI, Doty SB, Cardoso L, Fritton SP (2014) $3 \mathrm{D}$ assessment of cortical bone porosity and tissue mineral density using high-resolution micro-CT: effects of resolution and threshold method. J Bone Miner Res 29:142-150. CrossRef Medline

Ping J, Li N, Du Y, Wu X, Li L, Galbraith G (2007) Auditory evoked responses in the rat: transverse mastoid needle electrodes register before cochlear nucleus and do not reflect later inferior colliculus activity. J Neurosci Methods 161:11-16. CrossRef Medline

Rubel EW (1984) Ontogeny of auditory system function. Annu Rev Physiol 46:213-229. CrossRef Medline

Ruben RJ (1992) The ontogeny of human hearing. Acta Otolaryngol 112: 192-196. Medline

Sanchez-Calderon H, Rodriguez-de la Rosa L, Milo M, Pichel JG, Holley M, Varela-Nieto I (2010) RNA microarray analysis in prenatal mouse cochlea reveals novel IGF-1 target genes: implication of MEF2 and FOXM1 transcription factors. PLoS ONE 5:e8699. CrossRef Medline

Saunders JC, Doan DE, Cohen YE (1993) The contribution of middle-ear sound conduction to auditory development. Comp Biochem Physiol Comp Physiol 106:7-13. CrossRef Medline

Smith JT, Waddell BJ (2003) Developmental changes in plasma leptin and hypothalamic leptin receptor expression in the rat: peripubertal changes and the emergence of sex differences. J Endocrinol 176:313-319. CrossRef Medline

Souzanchi MF, Palacio-Mancheno PE, Borisov YA, Cardoso L, Cowin SC (2012) Microarchitecture and bone quality in the human calcaneus: local variations of fabric anisotropy. J Bone Min Res 27:2562-2572. CrossRef Medline 